\title{
LA ENFERMERÍA TRANSCULTURAL EN ESPAÑA
}

\section{Carmen Chamizo Vega}

Licenciada en Enfermería.

Principado de Asturias (España)

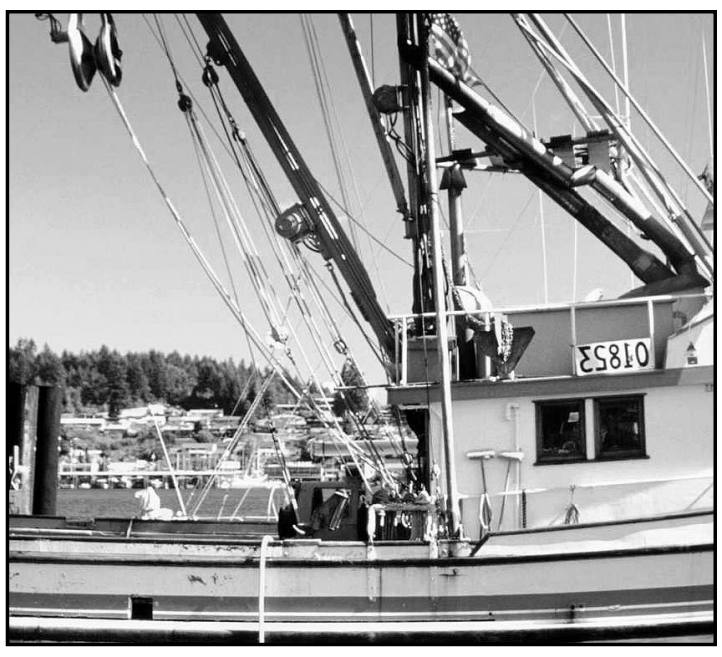

TRANS-CULTURAL NURSING IN SPAIN

\section{ABSTRACT}

$\mathrm{T}$ he present study analyzes the state of Trans-cultural Nursing in Spain, from a historical perspective. The outcome shows a lack of knowledge on these trans-cultural nursing theories at their basics although there are some nursing schools really advanced in the matter. In Spain at present, the question is some nursing schools are moving rather slow while others go too fast. Those who "think ahead" are not those in power to promote those theories and to put them into practice. Furthermore, our society is neither yet prepared to approach matters in a "non-medical way" nor yet organized to give an adequate feed-back to theory and practice care.

Key words: Trans-cultural nursing, nursing theories, competence, cultural

\section{RESUMEN}

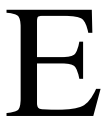

n este artículo se analiza la Enfermería Transcultural en España, desde la perspectiva histórica concluyendo que en dicho país, existe un desconocimiento de estas teorías en la base, pero sin embargo en ciertas escuelas existe un gran avance, es decir, que en España se va demasiado deprisa (unos) y otros demasiado lentos. Unos piensan y tienen ideas, pero no son los mismos que tienen poder para ponerlo en práctica, y además nuestra sociedad no está preparada para abordar temas "no médicalizados", ni tiene organizada la retroalimentación de la teoría y práctica de los cuidados.

Palabras clave: Enfermería Transcultural. Teorías de Enfermería. Competencia Cultural

\section{INTRODUCCIÓN}

Al leer el programa del Congreso, contacté con las personas de la mesa y comprobé que era disperso el discurso sobre los Modelos transculturales en España, debido quizás a lo novedoso de dichas teorías en dicho país. Comenté el tema con enfermeras expertas y su opinión fue que en España se va demasiado deprisa (unas) y otras demasiado lentas. Unas piensan y tienen ideas, pero no son las mismas que tienen poder para ponerlo en práctica, y además nuestra sociedad (y sistema sanitario ) no están preparados para abordar temas "no médicalizados”, ni se tiene organizada la retroalimentación de la teoría y práctica de los cuidados.

El por qué es diverso y revisando la bibliografía y desde una perspectiva histórica se entrevén algunas causas: la falta de asociacionismo en la base, la educación de enfermería en estos temas (que parte de 0 o menos 0 , tanto histórica como académicamente), sus docentes y su método (basado más en educar en valores que en enseñar a valorar y a participar), la forma de utilizar el poder (se enseña sumisión en las escuelas y ya sabemos que la que es sumisa nunca puede ser responsable y hacer aquello que se propone.). Además el poder masculinizado se adopta como forma de poder actual, gerencialmente hablando, existe una imposición de modelos frente a otros, en vez de coexistir de una manera articulada y adaptándose a auto- 
nomías e instituciones (políticas) y alimentados por los resultados es decir por los indicadores de gestión e investigación.

Mi objetivo en este artículo (1), ha sido aportar una visión de la situación en España de la Enfermería Transcultural, ser conciliadora en diálogo, hacer una propuesta de futuro y dar paso a distintos puntos de vista en el debate, para que se desarrollen los Modelos de Cuidados Culturales en España.

\section{ANÁLISIS DE LA SITUACIÓN EN ESPAÑA:}

Históricamente en España no existe una tradición asumida en cuanto a la utilización de Modelos de Enfermería y menos aún, si tratan temas tan novedosos como los de la Transculturalidad, Competencia Cultural o Cuidados Culturales, y se debe comenzar por hablar y debatir sobre ello, pues cada vez más son los que se preguntan: “¿Qué es eso?

Por otra parte los que más hablan sobre estos términos, son los que no tienen los pies puestos sobre la parte asistencial y sobre la realidad de la profesión, además la enfermera "de a pie" concibe lo de "cuidados culturales" como cosa de los "teóricos", porque no ha tenido una formación en esa línea.

Esta situación ha llevado a que no se empleen y se difundan estos Modelos en España, pese a que las migraciones juegan un papel fundamental en nuestro país.

Son diversos los Modelos de Enfermería Transcultural y Competencia Cultural que aparecen en la Bibliografía anglosajona, pero en las bases de datos españolas son escasos los artículos sobre el tema y sólo destaca la Revista de "Cultura de los Cuidados", donde se han publicado los modelos de Madeleine Leininger, el de Competencia Cultural de Larry Purnell y el Modelo de Tradiciones Culturales de Rachel Spector. También en las II Jornadas de Cultura de los Cuidados, celebradas el año 2003, en esta misma ciudad Dula Pacquiao, presentó su modelo o implicaciones éticas (también se pueden mencionar los de Camphina-Bacote y Papadopoulus, más conocidos en EEUU).

No obstante hay que decir, que los artículos existentes (2) de autores españoles, se centran en dar a conocer las teorías, sin considerar la aplica- ción práctica o las experiencias de la aplicación de estos modelos y sus resultados. Incluso podemos observar que aparecen diversas interpretaciones del término "transcultural" que no tienen nada que ver con lo que ahora hablamos.

Interesantes resultan los trabajos de: Lillo, Casabona, Galao y Mora sobre la aplicación de la Enfermería Transcultural en el contexto de la práctica clínica, concretamente trabajando de forma práctica-asistencial con la Etnoenfermeria de Leininger, las tradiciones en salud de Spector, la Competencia Cultural de Purnell, Papadopoulus, Meleis y la Ética cultural de Pacquiao. Estos trabajos se han centrado además en la calidad, satisfacción y percepciones desde el punto de vista de la Atención en Cuidados Culturales por parte de Enfermería, así como de la Gestión de dichos Cuidados Culturales. Destacar también el estudio descriptivo de Martínez sobre poblaciones socioculturales diversas que acuden al sistema sanitario público.

Se puede decir que en España, la Historia sobre la Enfermería Transcultural se reduce a los esfuerzos desde sus inicios por parte de la Asociación de Historia y Antropología de los Cuidados (3), que se ha ocupado en recopilar y trabajar estos modelos de Enfermería; publicando artículos y trabajos relacionados con dicha temática en la Revista Cultura de los Cuidados, organizando unas Jornadas anuales y acudiendo en la medida de lo posible a las Conferencias de la Transcultural Nursing Society.

\section{REFLEXIONES A TENER EN CUENTA EN EL DESARROLLO DE LA ENFERMERIA TRANSCULTURAL EN ESPAÑ}

\section{REFLEXIÓN FILOSÓFICA:}

El concepto de Enfermería Transcultural está basado en una ideología y sus modelos son como un marco de interpretación y evaluación de la realidad social referida a los cuidados. Su pretensión es orientar y justificar los cuidados enfermeros en las diferentes culturas y movimientos sociales.

Se puede decir que la ideología que la sustenta, es una ideología, con términos amplios y vagos y por tanto con falta de inserción gerencial o eficacia, en la "vida real". Es decir que por un lado hablamos de ideologías y teorías antropológicas, 
pero por otro, queremos insertar la Enfermería y los cuidados transculturales en un paisaje gerencial político (entendiendo política como algo que engloba todo). Estamos pues hablando de dos cosas: por un lado de un discurso con macroideologías y por otro de organizaciones que funcionan con eficacia y expertos.

Esto no es nuevo para la Enfermería, que desde su profesionalización ha ido construyendo teorías, como discursos legitimadores de las acciones que realizan las enfermeras; pero aún no hemos encontrado el puente entre el discurso-acción y el discurso-teórico. Incluso hoy día tenemos y estudiamos sofisticaciones discursivas (Diagnósticos de Enfermería), que acaban convirtiéndose en pseudoproblemas, e incluso necesitan sus propios expertos para poder abordarlos.

Esa tendencia a la sofisticación hace que exista, en algunas enfermeras, cierto desarme moral en la actividad concreta de cuidar (el rechazo de muchas enfermeras a ser superwoman y vivir por encima de sus posibilidades), y por tanto se puede esperar una pérdida de compromiso (si no les sirve esta teoría para su trabajo individual se desentienden de él; Ej.: ...eso era en la Escuela, ahora ya no...), que es paliada con recompensas individualizadas a través de grupos, donde la necesidad de pertenencia se ve cumplida y donde, si no persisten las recompensas individualizadas, tenderán a no persistir en su acción (...mira ahí va la trascultural....); y si persisten, serán por la incorporación de nuevas personas, quizás sin las ideologías iniciales, que van introduciendo modificaciones según sus propios intereses, lo que conlleva distorsiones a su vez.

Pero por otro lado, también hay que decir que, si nos expresamos con un discurso vago y cómodo, quizás no sea verdadero o no habrá manera de articularse con un discurso político gerencial solvente. Nos encontramos entonces ante un dilema: un discurso demasiado amplio y que no tenga inserción o un discurso complejo que no es capaz de llamar a la acción.

\section{ENFERMERÍA TRANSCULTURAL Y PODER:}

Antes de seguir, es preciso definir el término o concepto político de poder. Existen dos conceptos: el de Weber y otro el Francfortiano.
El primero define lo político como actividad gerencial de macroorganizaciones que gestionan lo público.

Sin embargo para la Escuela de Francfort que influye en toda la izquierda y el feminismo europeo y americano, lo político es todo (4).

Quizás esta última, sea la acepción que más hemos admitido en Enfermería, más que la gerencial, aunque en España por circunstancias de la Guerra Civil y las enseñanzas posteriores de la Dictadura, para las enfermeras (mujeres) la política no existía (5).

Esto quizás nos haga parecer una profesión difusa, que unido a la gran carga ideológica y a la ausencia de teorías en España, haya tenido problemas y tiene, a la hora de su inserción políticogerencial y en la medida que su espesor teórico se engrose sufrirá desencajes discursivos.

Pero pongamos que una de nosotras," enfermera trancultural”, llega a ministra de sanidad...¿qué sucedería ....cambiaría leyes, dimitiría a cargos y pondría a enfermeras transculturales en todos los puestos de gestión?. Creo simplemente que los temas gerenciales, no saben de teorías enfermeras y habría que abordarlos no con teorías o recursos enfermeros e ideológicos, sino con recursos expertos: este puede ser exigir un sistema de cuota de participación en un poder dado, por lo pronto en las estructuras que afecten a Enfermería y organizaciones relacionadas con problemas sociales y de salud.

\section{ENFERMERÍA TRANSCULTURAL Y CONOCIMIENTO}

Reflexionando sobre la cómo se trasmite el conocimiento, vemos que sólo acontece en comunidades de aprendizaje que presuponen una institucionalización, la presencia de algunas reglas, la adquisición de ciertos hábitos, el ejercicio de determinadas virtudes y la práctica de un esfuerzo compartido. Es decir, no sólo es esencial para la transmisión del conocimiento la sabiduría del maestro, sino también el deseo de saber del aprendiz; es decir, que lo importante no es enseñar sino aprender. Podríamos decir que por muchos modelos de cuidados transculturales que existan, sino hay enfermeras que los pongan en práctica y los cultiven y desarrollen, no se cumplirán los objetivos 
iniciales que dieron origen a estos Modelos Transculturales.

Así como el primer paso del maestro en la antigüedad, era darse cuenta del talento humano y la energía incomparablemente superior a la fuerza de la materia (capacidad de crear una obra frente a tornearla), en nuestra profesión también hay personas que cuidan y aprenden a su vez "prácticamente" de otras; y éstas no son sólo recursos, sino más bien fuentes de descubrimiento y generación de recursos (en La Odisea, no se le ocurre a Homero decir que Ulises era un recurso: nos muestra narrativamente cómo, en la empresa de su retorno a Ítaca, Ulises se manifestó como "fértil en recursos"). Es decir que "los maestros" deben de primar el factor humano y ver la práctica y a los que enseñan en ellas, como fuente de implementación de las teorías enfermeras.

Otro hecho destacable en España, es la formación del profesional de Enfermería, pasando de una eminentemente práctica a una teórica preponderante, con mucho énfasis en la Información (externa), pero no tanto en el Conocimiento (interno). Es decir que la Enfermería ha pasado en poco tiempo a dejar lo artesanal y enfocar sólo lo académico y creo que es muy importante la presencia de "maestros", más en el sentido en que atribuimos esta expresión a un "maestro albañil", a un "oficial" (como se decía en el medievo), que en la acepción que se adscribe ampulosamente a un famoso director de orquesta. Pero me refiero a maestros creativos y no soñadores (6).

También la Historia nos ha demostrado cómo se ha pasado de unos paradigmas a otros y que sobre lo que se sabía, se superponía el saber más y el nuevo conocimiento. Esto ocurrió en algunos aprendices con talento que se convirtieron en maestros y cuya orientación fue decir sin miedo, la veracidad de lo que creían.

\section{PROPUESTA DE FUTURO:}

Dicho lo anterior, realizo una pregunta: ¿qué hacer para que se desarrolle la Enfermería Transcultural y dichos Modelos en España?.

Tras el silencio y reflexión previos, quiero aportar varias respuestas e ideas que se me ocurren y que pueden dar lugar a otras más:

- Hay que promover mecanismos de horizontali- dad que hagan que las enfermeras transculturales se reconozcan en una causa y genealogía común, siendo como un gajo de una misma naranja, y demostrando que la Enfermería es de amplísimo arco social e histórico. Así se irá hacia delante con independencia de las adhesiones concretas e individuales a esta o aquella teoría (o modelo).

- La adhesión a la Enfermería Transcultural será muy difícil, si no existe la incentivación individual, representado por políticas concretas. Por qué no puede ser la Enfermería o los Cuidados Transculturales una rampa de salida hacia temas gerenciales y docentes (ámbitos de poder y salida para los licenciados en Enfermería).

- Llamar la atención en los profesionales de Enfermería, sobre la necesidad de recuperar la noción de sabiduría práctica, no lastrada por prejuicios; es decir crear un hábito de aprendizaje continuo de las personas, que fomente en las organizaciones los valores de la innovación y la solidaridad.

- El éxito de la organización de la Enfermería Transcultural en España, es el aumento del conocimiento enfermero en este campo (no sólo de la información) y ello está relacionado con las enfermeras y con su capacidad de pensar, en el sentido de discurrir, de pasar de unos conocimientos intelectuales a otros, es decir, de adquirir conocimientos nuevos. Pero este saber no es potestad de nadie y no es automáticamente transmisible, sino por medio de un aprendizaje. De ahí que lo que debe primar en la organización de la Enfermería Transcultural en el futuro, si queremos tener integración política y social - y por tanto éxito -, es un gran esfuerzo en la calidad de la educación, el aprendizaje y la investigación de estos temas.

- Un puente entre la teoría y la práctica debe ser la ayuda entre ambas y esto no supone que las jerarquías desaparezcan y sólo queden las REDES. Quiere decir que las jerarquías se establezcan en función del saber, en función de los lenguajes que cada uno domine y se conviertan así en protagonistas de una historia compartida: la de la Enfermería con las Culturas.

- La Ética constituye el fundamento y la orientación de toda sabiduría práctica y la confianza mutua, basada en la veracidad, es el límite que 
ninguna corporación o Asociación ha de vulnerar, porque entonces se haría internamente vulnerable. Este aspecto es importante desarrollarlo en la Enfermería Transcultural en España.

- No hay que perder de vista si se quiere desarrollar un plan de futuro, que la capacidad, potenciada por lo hábitos teóricos y prácticos, de llegar a saber cosas nuevas y aprender a realizarlas es lo que dará el índice de competitividad; es decir debemos conseguir que la Enfermería Española sea culturalmente competente con respecto a las teorías y la práctica en la Transculturalidad.

Todos estos planteamientos no quieren proponerles una "revolución", ya que creo que no existe ninguna fórmula mágica para que se desarrolle la Enfermería Transcultural en España, si no que hemos de estar buscando habitualmente soluciones cambiantes, procedimientos oportunos, respuestas operativas a las perplejidades que se nos plantean; perplejidades que ahora siempre son nuevas y cuya respuesta, por lo tanto, no se encuentra en ninguna parte: sólo se halla en el propio proceso de investigación práctica cuyo motor no es otro que el ejercicio implacable de la propia inteligencia.

\section{Agradecimientos: A Manuel Lillo e Isabel} Casabona por sus aportaciones. A José Siles y a J. A. Ávila por su deferencia al invitarme al Encuentro celebrado en Junio del 2004 en Alicante.

\section{BIBLIOGRAFÍA:}

- Andrews M, Boyle J, editores(1995). Transcultural Concepts in Nursing Care. $2^{\circ}$ edition. Philadelphia: Lippincott.

- Brink, P.J. (1999) Transcultural versus cross-cultural. Journal of Transcultural Nursing; 10(1), 6.

- Galao R.; Lillo M.; Casabona I.; Mora Mª D. (2005) ¿Qué es la enfermería transcultural? Una aproximación etimológica, teórica y corporativista al término. Evidentia enero-abril;2(4). En: http://www.index-f.com/evidentia/n4/99articulo.php [ISSN: 1697-638X].

- García M ${ }^{\mathrm{a}}$ I.; López M M N.; Vicente M $^{\mathrm{a}}$ J. (2003) Formación en relación del personal de enfermería. ¿Responde a una enfermería transcultural?. Index Enferm. invierno. XII(43): 88.

- Giger J, Davidhizar R. (1995) Transcultural Nursing: Assesment and Intervention. $2^{\text {a }}$ edition. St Louis: Mosby.

- González E, Comelles JMa . (2000) Psiquiatría Transcultural. Madrid: Asociación Española de Neuropsiquiatría.

- Leininger M.(1994) Nursing and Anthropology: Two Worlds to Blend. Columbus: Greyden Press.
- Leininger M. (1995) Transcultural Nursing. New York: McGraw-Hill.

- Lillo M.; Galao R.; Casabona I.; Mora Ma D.(2004) Competencia cultural y cuidados. Análisis conceptual y revisión bibliografica. Evidentia sept-dic;1(3). En: http://www.index-f.com/evidentia/n3/76articulo.php [ISSN: 1697-638X].

- Lipson J.G. (1999) Cross-cultural nursing: the cultural perspective. Journal of Transcultural Nursing. ; 10(1), 7.

- Luckmann J. (1999)Transcultural Communication in Nursing. New York: Delmar.

- Mariano, L.; Encinas, B. (2003) Cuidados y cuidadores transculturales en el Hospital Fernando Fonseca (Portugal). Index Enferm. Otoño. Año XII(42): 63-65.

- Meleis A.I. (2002) Cultural diversity research. En Western Institute of Nursing (Ed.). Silver threads: 25 years of nursing excellence; Denver, CO: Western Institute of Nursing.

- Montoya, R. (2003) Enfermería transcultural [comentario de: Las culturas de la salud. Spector, R.E. y Muñoz, M.J.]. Index Enferm. Invierno. Año XII (43): 85-86.

- Moreno, M.(2003) Inmigración hoy. El reto de los cuidados transculturales. Index Enferm. Otoño. Año XII(42)::29-33.

- Purnell L, Paulanka B, editores.(1998) Transcultural Health Care. Philadelphia: F. A. Company.

- Siles J., García E., Cibanal L., Galao R. (1999) La Enfermería Antropológica y Transcultural en el marco de la educación para el desarrollo. Una perspectiva histórica y epistemológica en el umbral del siglo XXI. Revista de Enfermería y Humanidades Cultura de los Cuidados; 6(3): 24-40.

- Siles J. (2003) Antropología y Enfermería. La necesaria simbiosis entre dos disciplinas para vertebrar culturalmente la teoría y la praxis de los cuidados. Index Enferm. Inv. Año XII(43):28-32.

- Spector R. (2000). Cultural Diversity in Health and Illness. 5th edition. Stamford: Appleton \& Lange.

(1) Artículo realizado sobre la ponencia expuesta en ALICANTE 16/6/04. Congreso de Enfermería Transcultural, por la autora .

(2) Búsqueda realizada en Medline y Cuiden ( Rev. Index de Enfermería y Evidentia):

(3) Creada por enfermer@s de la EUE de Alicante liderados por el profesor José Siles en 1996.

(4) Valcarcel, A. (1997)La política de las mujeres. Cátedra, pp100

(5) Discurso del Dr. Palanca a las enfermeras de Cruz Roja Española en 1916. Archivo Nacional de Cruz Roja Española

(6) La diferencia estriba en que los primeros saben cómo materializar sus ideas, cómo hacer operativos sus proyectos, lográndolo con naturalidad, pues quien domina un oficio posee una especie de empatía con la realidad sobre la que trabaja, de manera que es capaz de distinguir enseguida, lo esencial de lo accidental y saber cuál es el quid de la cuestión, eso que los anglosajones llaman the point. 